\title{
Overexpression of miR-21-5p as a predictive marker for complete tumor regression to neoadjuvant chemoradiotherapy in rectal cancer patients
}

Camila Miranda Lopes-Ramos ${ }^{1,2}$, Angelita Habr-Gama ${ }^{3,4}$, Bruna de Souza Quevedo5, Natlia Mariana Felcio 3,6, Fabiana Bettoni ${ }^{1}$, Fernanda Christtanini Koyama ${ }^{1}$, Paula Fontes Asprino ${ }^{1}$, Pedro Alexandre Galante ${ }^{1}$, Joaquim Gama-Rodrigues ${ }^{3,4}$, Anamaria Aranha Camargo ${ }^{1,5}$, Rodrigo Oliva Perez ${ }^{3,4,5}$ and Raphael Bessa Parmigiani ${ }^{*}$

\begin{abstract}
Background: Neoadjuvant chemoradiotherapy (nCRT) followed by radical surgery is the preferred treatment strategy for locally advanced rectal cancer. However, complete tumor regression is observed in a significant proportion of patients after nCRT, making them ideal candidates for alternative treatment strategies to this considerably morbid procedure. Identification of such patients based on clinical findings (complete clinical response - $C(R)$ is difficult mainly because it relies on subjective clinical and imaging studies. Our goal was to identify biomarkers capable of predicting complete response to nCRT.
\end{abstract}

Methods: We analyzed miRNA expression profile using deep sequencing in rectal tumor biopsies prior to nCRT. Differential expression was investigated by EdgeR for a training $(n=27)$ and a validation $(n=16)$ set of patients to identify miRNAs associated with treatment response (complete vs. incomplete). In vitro experiments with two cancer cell lines were also performed in order to evaluate the possible role of miRNAs on response to nCRT.

Results: We found 4 miRNAs differentially expressed between complete and incomplete responders to nCRT. In addition, validation was performed using an independent group of patients and miR-21-5p was confirmed as being overexpressed in complete responders. Overall sensitivity and specificity of miR-21-5p expression in predicting complete response to nCRT was $78 \%$ and $86 \%$ respectively. Interestingly, in a subset of patients with cCR followed by early local recurrence, the expression level of miR-21-5p was considerably low, similarly to incomplete responders. We also found SATB1, a miR-21-5p target gene and known multidrug resistance gene, whose expression was inversely correlated with miR-21-5p expression. Finally, we performed functional experiments and showed that miR-21-5p and SATB1 may be directly involved with poor response to nCRT in rectal cancer patients.

Conclusions: This study suggests miR-21-5p as a promising predictive biomarker, which should aid in the selection of patients with cCR to $\mathrm{nCRT}$ that potentially could be spared from radical surgery.

Keywords: miRNA, Predictive biomarker, Rectal cancer, miR-21-5p, SATB1, Chemoradiotherapy

\footnotetext{
* Correspondence: raphaparmigiani@gmail.com.br

'Centro de Oncologia Molecular, Hospital Srio-Libans, So Paulo, Brazil

Full list of author information is available at the end of the article
} 


\section{Background}

Treatment of locally advanced rectal cancer includes a combination of surgery and radiotherapy with or without chemotherapy (CRT and RT, respectively). In recent years, preoperative delivery of RT or CRT has become one of the preferred initial treatment options for the management of rectal cancer due to improved local disease control and significant tumor regression [1-3].

Tumor regression to neoadjuvant chemoradiation (nCRT) varies substantially among patients, and ultimately, complete pathological response ( $\mathrm{pCR}$ ) may develop in up to $42 \%$ of them [4]. These patients with pCR are associated with excellent oncological outcomes, particularly in terms of local disease control. Considering that radical surgery does not remove any residual cancer in these patients, alternative treatment strategies to this procedure have been considered in an effort to avoid unnecessary postoperative morbidity and mortality. A strategy of close observation without immediate surgery, known as Watch and Wait, has been proposed for highly selected patients with no clinically or radiologically detectable residual tumor (complete clinical response cCR) [5]. This strategy requires an intensive follow-up in which patients are submitted to frequent clinical, endoscopic and radiological assessments after completing nCRT [6].

However, clinical and radiological assessment of tumor response remains a significant challenge due to their subjectivity and inherent limitations of currently available studies. In this setting, identification of molecular markers capable of predicting complete response to nCRT would not only allow accurate selection of patients that benefit the most from nCRT but also identify ideal candidates to alternative treatment strategies without immediate radical surgery after achieving cCR.

miRNAs are small non-coding RNAs (18-25 nt) capable of regulating mRNAs post-transcriptionally by inducing their destabilization or translational repression [7]. miRNAs play important regulatory roles in several cellular processes such as cell proliferation, differentiation, and apoptosis. Changes in their expression profile have been reported for several types of cancer [8]. In fact, miRNAs may contribute to the tumorigenic process functioning as tumor suppressor genes or oncogenes depending on the genes they regulate $[9,10]$.

Importantly, different studies have also investigated how miRNA profiling may be associated with treatment response in different tumor types, motivating the search for their role as predictive molecular markers in cancer [11-13]. The goal of the present study was to compare miRNA expression profile of treatment-naive tumor biopsies derived from rectal cancer patients with complete or incomplete response to nCRT in order to identify specific miRNAs as predictive biomarkers.

\section{Methods}

\section{Patients and biological samples}

Patients with biopsy-proven rectal adenocarcinoma, cT2-4 N0-2 M0 located no more than $7 \mathrm{~cm}$ from the anal verge measured by rigid proctoscopy were eligible for the study. All patients were radiologically staged using high-resolution pelvic MRI, abdominal and chest CT scans and CEA levels. Patients with pregnancy or under the age of 18 were excluded from the study.

Endoscopic biopsies were taken from primary tumors, properly identified and snap frozen using liquid nitrogen prior to storage at $-80 \mathrm{C}$. Tumor fragments were verified for the presence of $\geq 80 \%$ viable cancer cells prior to RNA extraction using standard hematoxilin-eosin staining. Specimens with $<80 \%$ cancer cells were macrodissected to result in $\geq 80 \%$ of cancer cells.

This study was approved by the ethics committee from Hospital Alemo Oswaldo Cruz (Sao Paulo, Brazil reference number 19/08). Informed consent was obtained from all study participants prior to sample collection.

\section{Treatment and assessment of response}

All patients underwent nCRT as described elsewhere [5]. Briefly, nCRT consisted of 50.4-54Gy of radiation and concomitant 5FU-based chemotherapy. Patients were clinically reassessed for tumor response at least 12 weeks from nCRT completion using digital rectal examination, rigid proctoscopy, pelvic MRI and CEA levels [6]. Patients with evidence of residual disease (incomplete clinical response) such as residual ulcers, irregularity, mass or stenosis at clinical examination were referred to immediate radical surgery. Likewise, patients with radiological evidence of residual disease including the presence of nodal metastases or residual primary cancer were also referred to radical surgery. Patients with clinical, endoscopic or radiological evidence of $\mathrm{CCR}$ were recommended no immediate radical surgery and were enrolled in a strict follow-up program (Watch \& Wait strategy) [5].

\section{Groups for comparison}

Based on chronological order, the first patients matching our criteria were included in the training set (27 samples) and the samples collected later on were included in the validation set (16 samples). Patients were grouped according to response to nCRT based on clinical and pathological findings. The complete responders group included patients with clinical evidence of complete response (cCR) sustained for at least 24 months of follow-up and patients with complete pathological response (pCR) who underwent radical surgery due to inability to rule out residual disease. The incomplete responders group included patients with significant residual disease detected after pathological examination of the resected specimen, including ypT2-4 or ypN+ 
and $>10 \%$ residual cancer cells (tumor regression grades TRG0-2 according to Dworaks classification [14]. Therefore, patients with incomplete but with near-complete response including ypT1N0 or $\leq 10 \%$ residual cancer cells (TRG3) were excluded from the study. Differential miRNA expression analyses were performed comparing the complete to incomplete responders.

\section{miRNA sequencing analysis}

Total RNA was extracted with Trizol reagent (Invitrogen), and RNA quality was evaluated on 2100 Bioanalyzer (Agilent). All samples had RNA integrity number (RIN) above 6. Total RNA $(10 \mu \mathrm{g})$ was enriched for small RNAs (up to $250 \mathrm{bp}$ ) using PureLink miRNA Isolation Kit (Invitrogen). miRNA libraries were prepared using SOLiD Total RNA-Seq Kit (Life Technologies), according to the manufacturers recommendations and were sequenced on SOLiD4 (Life Technologies) in 35 bp single read runs.

Sequencing data was analyzed using the CLC Genomics Workbench 5.1 software (CLC Bio). Initially, sequencing adapters were trimmed from sequencing reads. The remaining sequences were mapped against the human miRNA database, miRBase (www.mirbase.org, release 18) $[15,16]$, allowing one mismatch. At last, known mature miRNAs were annotated according to miRBase and the number of sequences for each miRNA was used for differential expression analysis. Normalization was done by dividing the number of sequences of a certain miRNA by the total number of miRNA mapped sequences for a given sample and multiplying by one million. This resulted in a value corresponding to the number of sequences (counts) per million (cpm) for each miRNA. After normalization, low expressed miRNAs were filtered from further analysis to increase detection power of the statistic tests. Only miRNAs with a minimum of $20 \mathrm{cpm}$ in at least seven samples were kept in the analyses (Additional file 1: Table S1).

Differential expression between the two groups of patients was performed with EdgeR (version 2.6.7) [17] available on Bioconductor 2.10 [18]. P-values were adjusted for multiple testing using the Benjamini and Hochberg method to control the false discovery rate (FDR) [19]. Only miRNAs with FDR under 0.05 were considered as differently expressed.

\section{Clustering and response prediction analysis}

Hierarchical clustering and bootstrap statistical analysis were performed using the functions heatmap and pvclust available in the $\mathrm{R}$ statistical software (version 3.0.1). These analyzes were based on Euclidean distance and average linkage clustering using cpm normalized data. Principal Component Analysis (PCA) was performed using the Matlab language function princomp (version R2009b). PCA was carried out by eigenvalue decomposition of the covariance matrix after mean centering the data matrix for each attribute. The first principal component (i.e., the direction along which miRNAs expression show the largest variance) and the second principal component (i.e., the next best direction uncorrelated with first one) were retrieved and plotted to illustrate similarities between samples.

Receiver operating characteristic (ROC) curve for each miRNA was generated using cpm values obtained from RNA-Seq data, using GraphPad Prism software (version 4.03). To determine accuracy to predict nCRT, the area under the ROC curve (AUC) was calculated. The cutoff expression values (cpm) were defined in order to maximize the number of correctly classified samples. Finally, sensitivity and specificity based on this cutoff were calculated.

\section{miR-21-5p putative target genes}

We initially used TargetScan software (www.targetscan. org) to search for predicted miR-21-5p targets [20]. We considered only conserved sites in conserved target genes. Using whole transcriptome sequencing data from 19 of the primary rectal tumors used in this study (unpublished data), we searched for the expression of predicted targets that were inversely correlated (Pearson correlation) to miR-21-5p expression using GraphPad Prism software (version 4.03).

Further exploring whole transcriptome data, SATB1 gene expression was compared between incomplete responders $(n=13)$ and complete responders $(n=6)$. Mann Whitney test was performed using GraphPad Prism software (version 4.03).

\section{Validation of miR-21-5p and SATB1 expression by qPCR} miR-21-5p and SATB1 gene expression was evaluated through qPCR in 10 and 11 samples, respectively (the samples that were used for validation of SATB1 were the same as the samples for miR-21-5p plus another sample from an incomplete responder that still had RNA available). For miR-21-5p, the QIAGEN miScript PCR system was used. cDNA was generated with miScript II RT kit (QIAGEN) and $3 \mathrm{ng}$ were used as template in each qPCR reaction. miR-21-5p miScript primer assay and miScript SYBR Green PCR kit were also used. Reactions were performed in triplicates using 7900HT Fast System (Applied Biosystems). Based on our miRNA-Seq data, we chose miR140-5p and miR-224-5p to be used for normalization of qPCR experiments since they were most stably expressed miRNAs among our samples [21,22].

SATB1 gene expression was evaluated with GoTaq qPCR Master Mix (Promega) using the following primers: SATB1Fw: GGTACAAACATTTCAAGAAGAC and SATB1Rev: CATGATTGGCGCCTTGCT. PUM1 and HMBS genes were used for normalization (PUM1Fw: TGTACTT 
ACGAAGAGTTGCGATGTG PUM1Rev: CCAGGCCAG CGGAAGAT; HMBSFw: GGCAATGCGGCTGCAA HM BSRev: GGGTACCCACGCGAATCAC).

Relative expression was calculated based on $\triangle \Delta C T$ method using a colorectal cancer cell line (HCT116) as reference sample [23]. Mann Whitney test was performed using GraphPad Prism software (version 4.03).

\section{Clonogenic assays}

Colorectal tumor cell lines, HCT116 and SW480, were obtained from American Type Culture Collection (ATCC), and cultured following their recommendations.

Cells were plated at a low density (500 cells/well) in 4 wells plates $\left(1.9 \mathrm{~cm}^{2}\right.$ well area) and transfections were carried out with Lipofectamine RNAiMAX Transfection Reagent (Invitrogen). HCT116 were transiently transfected with $30 \mathrm{nM}$ mirVana miRNA Inhibitors (hsa-miR-21-5p or inhibitor control) and SW480 were transfected with 10 nM mirVana miRNA Mimics (hsa-miR-21-5p or mimic control) (Life Technologies). $24 \mathrm{~h}$ after transfection, cells were treated with $5 \mu \mathrm{M}$ 5-fluorouracil (5-FU, Libbs) and irradiated with 1 Gy dose for HCT116 and 2Gy for SW480 using GammaCell 3000 equipment (Elan). After 12 days, colony formation was quantified. Cells were fixed with formaldehyde, stained with crystal violet and air-dried. For colony quantification, the dye from stained cells was solubilised with $10 \%$ acetic acid and absorbance was measured at $595 \mathrm{~nm}$ using Infinite 200 PRO (Tecan Group Ltd). Two independent experiments were performed in quadruplicates for each cell line.

\section{Results}

Training and validation sets of patients

Clinical and demographics features of all 43 patients included in this study are summarized in Table 1. Overall, 27 patients were analyzed in the training set, including 7 complete responders and 20 incomplete responders. In addition, 16 patients were analyzed as a validation set, being 7 complete responders, 5 incomplete responders and 4 patients that initially presented $\mathrm{CCR}$ but developed early local recurrence (within the first 16 months of follow-up after nCRT).

\section{miRNA differential expression to predict nCRT}

Primary tumor biopsies were collected before nCRT and used for small RNA sequencing. On average, 37 million sequences were generated for each sample. After mapping and annotation, 711 mature miRNAs were detected per sample. The number of sequences for each specific miRNA was used to estimate its expression, and low

Table 1 Clinical and demographics data of patients included in the study

\begin{tabular}{|c|c|c|c|c|c|c|c|c|}
\hline & \multicolumn{3}{|l|}{ Training set } & \multicolumn{5}{|c|}{ Validation set } \\
\hline & $\begin{array}{l}\text { Complete } \\
\text { responders }\end{array}$ & $\begin{array}{l}\text { Incomplete } \\
\text { responders }\end{array}$ & $\bar{p}$ & $\begin{array}{l}\text { Complete } \\
\text { responders }\end{array}$ & $\begin{array}{l}\text { Incomplete } \\
\text { responders }\end{array}$ & Recurrence & & $p$ \\
\hline $\mathrm{N}$ & 7 & 20 & & 7 & 5 & 4 & & \\
\hline Age (years) & 58.910 .9 & 54.213 .7 & ns & 60.413 .6 & 59.614 .5 & 69.315 .0 & ns & \\
\hline Gender (M/F) & $2 / 5$ & $9 / 11$ & ns & $3 / 4$ & $3 / 2$ & $3 / 1$ & & ns \\
\hline Distance anal verge $(\mathrm{cm})$ & 4.02 .6 & 4.32 .2 & ns & 4.62 .4 & 2.22 .8 & 40.8 & ns & \\
\hline Tumor size $(\mathrm{cm})$ & 4.40 .8 & 4.61 .5 & ns & 3.60 .9 & 51.9 & 51.4 & ns & \\
\hline Initial staging & & & ns & & & & & ns \\
\hline $\mathrm{T} 2$ & 0 & 2 & & 4 & 2 & 0 & & \\
\hline T3 & 7 & 17 & & 2 & 2 & 4 & & \\
\hline T4 & 0 & 1 & & 1 & 1 & 0 & & \\
\hline No & 4 & 7 & & 2 & 2 & 2 & & \\
\hline $\mathrm{N}+$ & 3 & 13 & & 5 & 3 & 2 & & \\
\hline \multicolumn{9}{|l|}{ Staging after CRT } \\
\hline $\mathrm{CCR}$ & 3 & - & & 6 & - & 4 & & \\
\hline $\mathrm{pCR}$ & 4 & - & & 1 & - & - & & \\
\hline урт2 & - & 6 & & - & 1 & - & & \\
\hline урT3 & - & 13 & & - & 4 & - & & \\
\hline урT4 & - & 1 & & - & 0 & - & & \\
\hline ypNO & - & 12 & & - & 1 & - & & \\
\hline ypN+ & - & 8 & & - & 2 & - & & \\
\hline ypNx & - & 0 & & - & 2 & - & & \\
\hline
\end{tabular}


expressed miRNAs were removed in order to increase detection power of statistic tests. Finally, we performed differential expression analysis using, on average, 330 mature miRNAs for each sample (Additional file 1: Table S1).

The first approach to identify molecular markers capable of predicting tumor response to nCRT was to perform differential miRNA expression analysis using the 27 samples from the training set. Comparison between complete and incomplete responders showed 36 differently expressed miRNAs $(\mathrm{p}<0.05)$. In order to apply a more stringent control over false positives, $\mathrm{p}$-values were adjusted for multiple testing, which controls false discovery rate (FDR) [19]. Thus, 4 miRNAs had a FDR $<0.05$ and were considered as reliable differentially expressed when comparing complete to incomplete responders (Table 2). Three miRNAs were overexpressed in complete responders (miR-21-5p, miR-1246, and miR1290-3p) and one, miR-205-5p, was overexpressed in incomplete responders.

After determining differentially expressed miRNAs, we evaluated their efficiency in discriminating complete responders from incomplete responders patients. First, we performed hierarchical clustering analysis which resulted in two major groups, one containing only incomplete responders and the other one with all complete responders plus 3 incomplete responders (Figure 1A). Second, the expression level (given in count per million, cpm) of the 4 differentially expressed miRNAs was used to perform Principle Component Analysis (PCA). This analysis revealed good separation between complete and incomplete responders (Figure 1B). Consistent with the hierarchical clustering analysis, the same 3 incomplete responders showed greater similarity with patients from the complete responders group.

\section{miRNAs as potential predictive markers}

We have also investigated the potential of each of the 4 differently expressed miRNAs to be individually used as a predictive biomarker. In order to estimate the efficiency on correctly classifying patients according to treatment response, we calculated the area under the ROC curve (AUC) for each miRNA. We found an AUC of $0.94,0.74,0.70$, and 0.63 for miR-21-5p, miR-1246, miR-1290-3p, and miR-205-5p respectively (Figure 2).
Also, the expression cutoff point was determined for each miRNA to maximize sensitivity and specificity. Considering the normalized expression values (in cpm) obtained by RNA-Seq, the established cutoff for each miRNA resulted in a sensitivity and specificity of $100 \%$ and $85 \%$ for miR-21-5p, $86 \%$ and $65 \%$ for miR-1246, $71 \%$ and $75 \%$ for miR-1290-3p, and $86 \%$ and $55 \%$ for miR-205-5p, respectively (Figure 2). These results suggested miR-21-5p as the best putative predictive marker.

\section{Validation of miRNA differential expression in independent samples}

In order to verify whether the differential expression of these miRNAs was not limited to the training set of samples, we have also analyzed a validation set of samples, including 7 complete responders and 5 incomplete responders. miRNAs libraries from these primary tumors were sequenced and global differential expression analysis between complete and incomplete responders was performed. Thus, we found miR-21-5p as a common differently expressed miRNA ( $p$-value $<0.01$ ). The other 3 miRNAs did not show differential expression in the validation set and therefore, were not used in the following analyses.

To further validate miR-21-5p as a predictive marker for nCRT, we also analyzed its expression in samples from patients that initially presented cCR to nCRT and were spared from immediate surgery but during follow up visits, presented early local recurrence requiring salvage resection (named here as early recurrence group). These patients most definitely had clinically undetectable residual disease after nCRT. Noteworthy, miR-21-5p expression pattern in these patients was very similar to incomplete responders (Figure 3). More importantly, miR-21-5p expression in these two groups of patients (incomplete responders and early recurrence group) was significantly lower than the complete responders group (p-value <0.01). These results indicated that although physicians could not distinguish between patients who presented bona fide complete response and patients who initially presented complete clinical response but faced early local recurrence, they have tumors with important molecular differences, including miR-21-5p expression.

Table 2 Differentially expressed miRNAs

\begin{tabular}{llllr}
\hline miRNA & $\begin{array}{l}\text { Complete responders } \\
\text { mean expression }(\mathbf{c p m})\end{array}$ & $\begin{array}{l}\text { Incomplete responders } \\
\text { mean expression } \mathbf{( c p m})\end{array}$ & Fold change & P-value \\
\hline miR-205-5p & 3.67 & $2,099.32$ & 0.002 & $6.21 \mathrm{E}-06$ \\
miR-21-5p & $106,020.55$ & $31,438.53$ & 3.372 & $1.99 \mathrm{E}-04$ \\
miR-1290-3p & 115.14 & 30.02 & 3.835 & 0.002 \\
miR-1246 & 65.58 & 19.60 & 3.346 & 0.028 \\
\hline
\end{tabular}

miRNAs mean expression is shown for each response group to nCRT and fold change of complete responders relative to incomplete responders. Statistical significance can be verified by P-values and false discovery rate (FDR). 

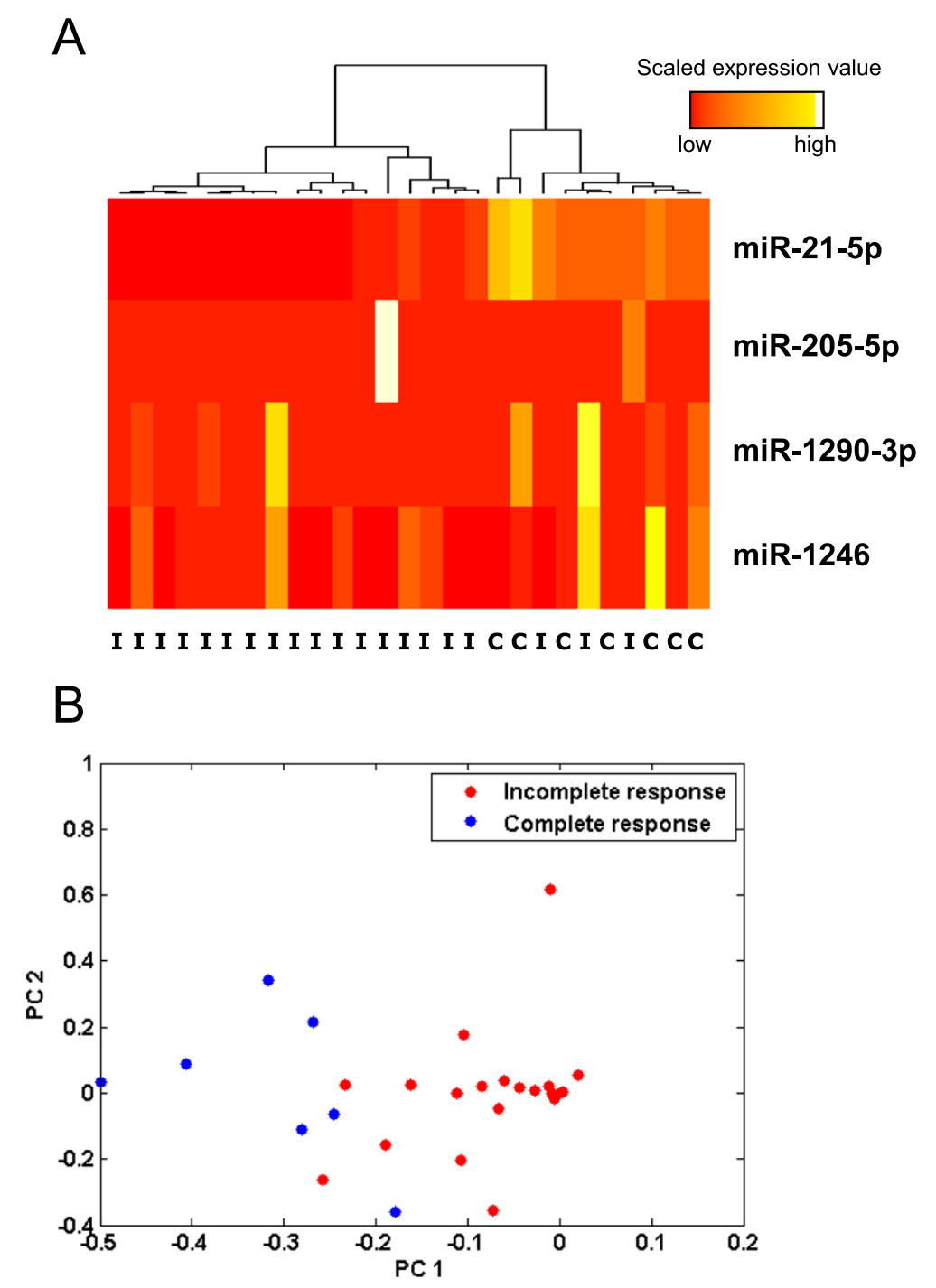

Figure 1 Patients clustering based on the expression of 4 differently expressed miRNAs. A) Hierarchical clustering heatmap for incomplete (I) and complete responders (C). B) PCA was conducted for 27 samples and 4 variables (differently expressed miRNAs). Blue dots: complete responders; red dots: incomplete responders.

In addition, we determined the overall predictive power of miR-21-5p expression using the cutoff established on the ROC curve for the training set $(70,000 \mathrm{cpm})$ and all the samples included in the study (from training and validation set). For this estimation, we considered the early recurrence group as incomplete responders. Thus, miR21-5p expression presented $78.5 \%$ (11/14) sensitivity and $86 \%(25 / 29)$ specificity to identify patients with complete response to nCRT. Similarly, miR-21-5p expression also showed good positive $(73 \%, 11 / 15)$ and negative predictive values $(89 \%, 25 / 28)$.

Finally, we have also validated the miR-21-5p differential expression by qPCR, a more easily and cheaper implemented method compared to deep-sequencing. Since our samples were all pre-treatment rectal tumor biopsies, they yielded a limited amount of RNA usually completely used for miRNA library construction. Therefore, for qPCR validation, we only tested 10 samples that still had RNA available (3 complete responders and 7 incomplete responders). The higher miR-21-5p expression in complete responders was once again validated (Additional file 2: Figure S1). However, possibly due to the small number of samples tested, this difference was not statistically significant ( $\mathrm{p}$-value $=0.067$ ).

We have also investigated whether miR-21-5p expression was associated with clinical and pathological parameters. In order to do that, we categorized samples into two groups: high miR-21-5p expression (expression above the established cutoff, 70,000 cpm) and low miR21-5p expression. No statistically significant association 


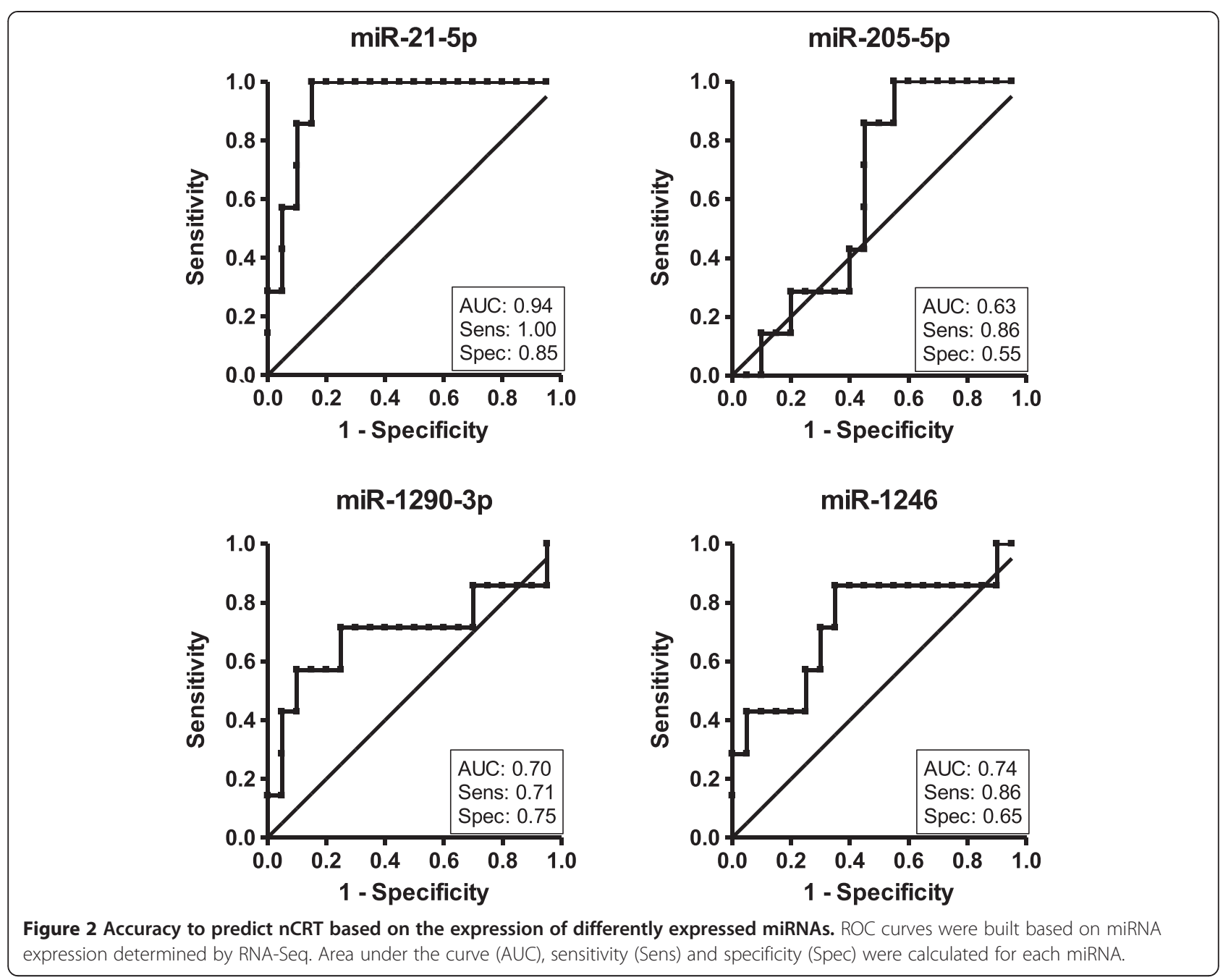

was found between miR-21-5p expression and any of the parameters analyzed, such as age, gender, tumor size, tumor distance from anal verge, and initial $\mathrm{T}$ and $\mathrm{N}$ staging (Additional file 3: Table S2).

\section{Potential role of miR-21-5p on treatment response}

In order to investigate whether miR-21-5p expression is directly involved in treatment response, we analyzed the expression pattern of its putative target genes using whole transcriptome RNA-Seq data from 19 samples of the training set patients (6 complete responders and 13 incomplete responders unpublished data). Using TargetScan software [20], we found 307 predicted miR21-5p targets genes with high prediction score. Among them, we were able to detect the expression (measured in reads per kilobase of transcript per million reads mapped, RPKM) for 249 genes in the 19 patients. Considering the negative regulation of miRNAs over their target genes expression, we searched for predicted targets for which the expression pattern was inversely correlated to miR-21-5p expression. Using this approach, we only found a significant negative correlation $(r=-0.5$ and $\mathrm{p}$-value $=0.03$ ) for SATB1 gene (Figure $4 \mathrm{~A}$ and Additional file 4: Table S3). This is a global gene regulator that has been reported to confer malignant behavior and multidrug resistance (MDR), as well as being associated with poor prognosis in several cancer types, including rectal cancer [24-26]. Even though other authors have shown that miR-21-5p can regulate the expression of other target genes such as PTEN, MSH2, Cdc25A, SPRY2 and PDCD4, and therefore be associated with worse response to therapy in other tumor types, we did not observe a significant inverse correlation between the expression of these target genes and of miR-21-5p in our samples (Additional file 3: Table S4) [27-31].

It has already been shown that miR-21-5p can negatively regulate SATB1 expression [32,33]. Indeed, we have confirmed such regulation in vitro by transiently manipulating miR-21-5p expression in 2 different colorectal cancer cell lines. HCT116 and SW480 cells were chosen because they differ significantly on miR-21-5p expression levels: 5-fold higher in HCT116 cells (Figure 5A). Thus, we have 


\section{miR-21-5p}

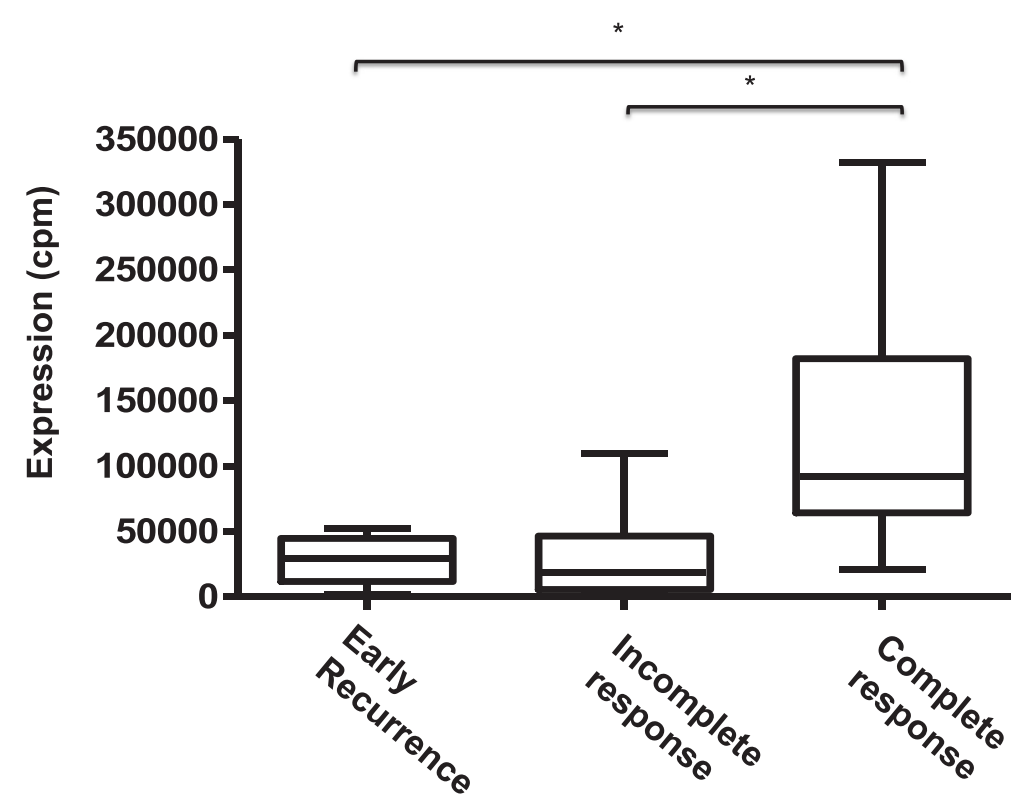

Figure 3 miR-21-5p expression in all samples included in the study. Patients were divided in 3 groups according to nCRT response: patients with initial cCR followed by early local recurrence $(n=4)$, incomplete responders $(n=25)$, and complete responders $(n=14)$. miR-21-5p expression is given as normalized values (cpm) from RNA-Seq data. Statistical analysis was performed with EdgeR (*FDR $<0.05)$.

transfected SW480 cells with miR-21-5p mimic and observed by qPCR a reduction on SATB1 mRNA expression (Figure 5B). Accordingly, we have knockeddown miR-21-5p expression in HCT116 cancer cells and observed an increased expression of SATB1 (Figure 5C). These results confirmed the negative regulation of miR-21-5p on SATB1 expression and corroborated the negative correlation of their expression observed in our patients samples.
Further exploring the whole transcriptome data from these 19 patients, we found a significantly higher SATB1 expression in incomplete responders compared to complete responders, according to our hypothesis $(\mathrm{p}$-value $=0.01$ ) (Figure 4B). In addition, we have also evaluated SATB1 expression by qPCR in 11 primary tumors ( 3 complete and 8 incomplete responders). A higher SATB1 expression in incomplete responders was once again observed. However, similar to what we observed for miR-21-5p, this
A

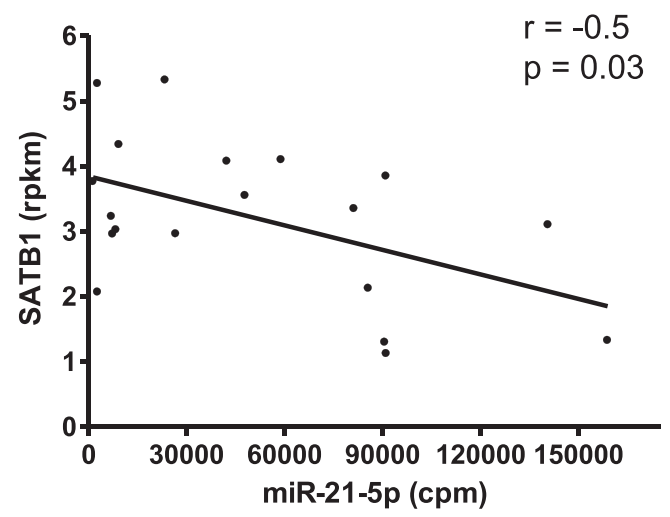

B

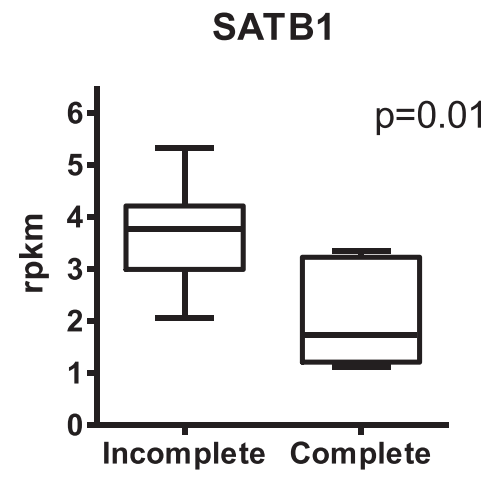

Figure 4 Expression analysis of SATB1 gene. A) Correlation between SATB1 and miR-21-5p expression, measured by RNA-Seq, in the same primary rectal tumors. r: Pearson correlation factor. B) SATB1 expression according to $n C R T$ response: incomplete responders $(n=13)$, and complete responders $(n=6)$. SATB1 expression is given as normalized values (rpkm). Mann Whitney test performed. 


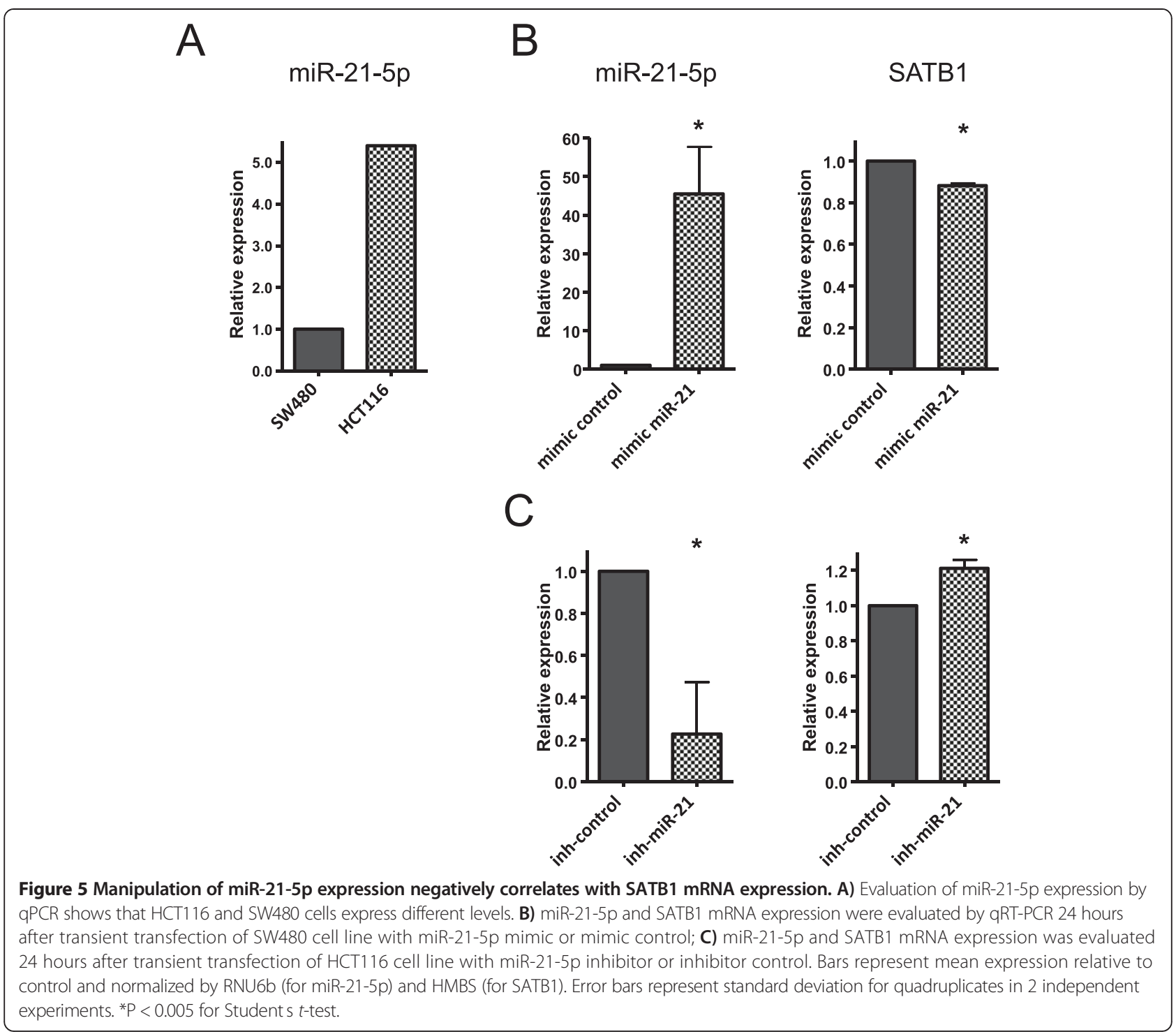

difference was not statistically significant $(\mathrm{p}$-value $=0.13)$ (Additional file 2: Figure S2). These results suggest SATB1 as a potential candidate for functional studies, which could elucidate the possible direct involvement of miR-21-5p in response to $\mathrm{nCRT}$ in rectal cancer patients.

miR-21-5p and SATB1 expression in colorectal cancer cells is directly involved with treatment response

Further exploring the involvement of miR-21-5p on response to nCRT in rectal cancer patients we have challenged HCT116 and SW480 colorectal cancer cell lines to a similar treatment in vitro. In order to properly evaluate the role of miR-21-5p, we have transiently manipulated its expression in both of them and performed clonogenic assays to verify whether there were changes on their sensitivity to the chemoradiation treatment. Experiments were performed with cells cultured under control conditions as well as submitted to one dose of radiation combined with 5-FU treatment (see details in Methods).

Firstly, SW480 cells were transiently transfected with miR-21-5p mimic and mimic control, and submitted to the clonogenic assay. As expected, miR-21-5p increased expression resulted in decreased SATB1 expression and also turned SW480 cells more sensitive to chemoradiation treatment (Figure 6A). Similarly, HCT116 cells, which express higher levels of miR-21-5p, were transiently transfected with mir-21-5p inhibitor and inhibitor control, and submitted to the clonogenic assay. Inhibition of miR-21-5p expression resulted in an increased SATB1 expression and decreased sensitivity to chemoradiation treatment (Figura 6B). According to our findings 


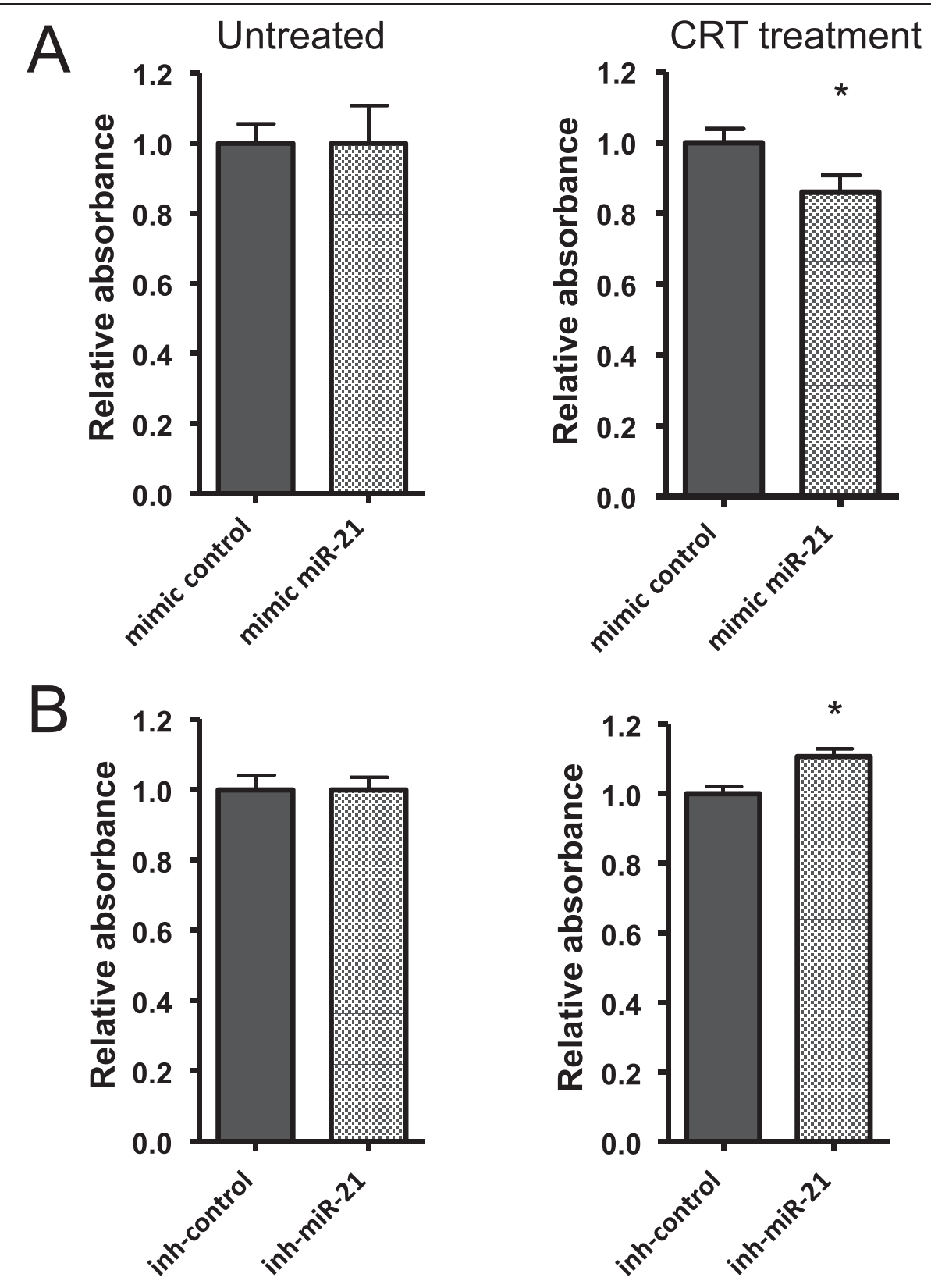

Figure 6 Manipulation of miR-21-5p expression alters response to chemoradiation in vitro. A) Quantification of colony formation assays for SW480 cell line transfected with miR-21-5p mimic or mimic control. B) Quantification of colony formation assays for HCT116 cell line transfected with miR-21-5p inhibitor or inhibitor control. Cells were grown under control conditions or submitted to CRT treatment, which consisted of $5 \mu \mathrm{M}$ 5-FU and a single dose of 2Gy (A) or 1Gy (B) radiation. Graphs show relative absorbance of colonies stained with crystal violet, normalized to untreated controls. All values are presented as means s.d. relative to mimic or inhibitor controls from two independent experiments with quadruplicates. ${ }^{*} \mathrm{P}<0.005$ for Students $t$-test.

in patients, these results suggest that, through regulation of SATB1 expression, miR-21-5p is directly involved on response to chemoradiotherapy.

\section{Discussion}

Standard treatment of locally advanced rectal cancer may include nCRT followed by radical surgery in most patients. However, the observation of PCR in up to $42 \%$ of patients undergoing $\mathrm{nCRT}$ has challenged the role of radical surgery in this setting and alternative treatment strategies for these patients have been suggested $[4,5,34]$. Identification of these patients by clinical and imaging assessments may be very difficult and remains restricted to specific dedicated centers. Therefore, most of these patients still undergo potentially unnecessary radical surgery including total mesorectal excision. Molecular 
markers capable of accurately predicting tumor response to nCRT would be of great clinical significance since these patients could be spared from radical surgery and from morbidities associated with this procedure.

Previous studies searching for predictive molecular markers in rectal cancer patients have used microarray and qPCR to evaluate mRNA or miRNA profiling [35-42]. However, patients response classification varied considerably among these studies. The Responders group frequently included complete pathological responders and partial responders (based on T-level downstaging or tumor regression grades). In addition, although it has been shown that $\mathrm{pCR}$ rates may considerably increase up to 12 weeks after nCRT, most of these studies have assessed tumor response at considerably short intervals from nCRT (most frequently after 6 weeks) $[35,37,38,43]$. Thus, comparison between studies may be quite difficult and rather inconclusive. Regarding miRNA profiling, three recent studies have identified signatures with good accuracy to predict nCRT response [40-42]. However, none of them validated their signatures in an independent group of patients. Importantly, comparing the molecular signatures, there is no overlapping miRNA among them, suggesting that these signatures might be characteristic of the samples used in each study.

To the best of our knowledge, this is the first study to perform global miRNA sequencing followed by differential expression analysis of primary rectal tumor samples, and to use, as complete response group, samples from patients with $\mathrm{pCR}$ as well as with sustained $\mathrm{CCR}$ to nCRT. In addition, the exclusion of patients with nearcomplete responses rather than including them in complete or incomplete responder groups allowed us to compare only patients/tumors with significant clinical discrepancies in their outcomes (response to nCRT), favoring the identification of molecular differences. Using this approach, we identified 4 differently expressed miRNAs between complete and incomplete responders in the training set. Based on their expression and by performing two different analyses (hierarchical clustering and PCA), samples could be clustered into two distinct groups. Importantly, in all of them, all 7 complete responders were clustered together. The predictive power of each miRNA was also estimated through ROC curves and miR-21-5p showed the best accuracy $(\mathrm{AUC}=0.94)$.

Due to clinical and molecular heterogeneity of tumor samples, we expect that predictive markers found in one group of samples might not be valid for a different group of samples. Thus, miRNA differential expression was further evaluated in additional samples. Among the 4 miRNAs, miR-21-5p maintained a significant difference in expression levels, being overexpressed in patients with complete response to nCRT, and showing the potential of this miRNA to be used as a predictive marker. Interestingly, in all patients with initial cCR followed by early local recurrence, miR-21-5p showed an incomplete response expression pattern. Thus, if miR-21-5p expression was used to help physicians to decide whether to operate these patients or not, they would have been correctly submitted to surgery immediately after the resting period post-nCRT.

When used as a predictive marker on all samples included in the study, miR-21-5p expression showed high sensitivity (78.5\%) and specificity (86\%) on the identification of complete responders. Among complete responders, it is important to note that miR-21-5p expression was not significantly different between pCR and cCR. Importantly, considering only pCR patients, 4 out of 5 (80\%) were correctly predicted based on miR-21-5p expression. These patients were submitted to surgery due to inability to rule out residual disease by clinical and radiological exams, suggesting again that a high percentage of patients could benefit from such biomarker and be spared from radical surgery. Although we have studied a limited number of samples, this is the first study that has used a validation set of samples to confirm the differential expression observed in a training set. Thus, miR-21-5p emerges as a promising predictive marker to nCRT that still needs to be further validated in a larger cohort.

miR-21-5p is overexpressed in different tumor types, including colorectal cancer (CRC), possibly acting as an oncogenic miRNA with important roles in cell proliferation, apoptosis and invasion [44-46]. Therefore, miR-21$5 \mathrm{p}$ has been considered a promising diagnostic/prognostic biomarker [47-49]. However, the literature is controversial regarding miR-21-5p expression and treatment response. Although several in vitro studies using CRC cell lines have shown that overexpression of miR-21-5p induces resistance to chemotherapy [30,31,50], few studies evaluated miR-21-5p expression in CRC primary tumors and in the context of radiotherapy. In one of these studies, patients treated with adjuvant chemotherapy, high miR-21-5p expression predicted worse overall survival, suggesting an association between high miR-21-5p expression and poor therapeutic outcome [51]. However, comparison between these results and ours is not straightforward, since they investigated miR-21-5p expression in the context of colon adenocarcinoma, adjuvant chemotherapy and long-term survival outcomes. All of these discrepancies lead to inherent limitations in comparisons between studies and possibly yielding controversial findings.

In one of the few studies with rectal cancer patients submitted to nCRT, miR-21 expression was evaluated in macrodissected tumor tissue before and after treatment, and in normal rectal tissue from resection specimen [52]. There was no significant difference on miR-21 expression level between pre and post-treatment tumor samples as well as between post-treatment tumor and normal tissues. 
miRNAs functions are usually inferred through the identification of their target genes. Considering the negative regulation imposed by miRNAs it can be assumed that they perform an opposite role of their targets. Searching for the expression of miR-21-5p targets in our samples, we found an interesting negative correlation for SATB1 gene and miR-21-5p expression. As expected, when we compared SATB1 expression among complete and incomplete responders, a significantly higher expression was observed in incomplete responders. SATB1 is a chromatin modifier and it has also been shown to be a MDR gene in several cancer types [25,53,54].

As mentioned, although other authors have shown that miR-21-5p can regulate the expression of other target genes such as PTEN and MSH2, and therefore be associated with worse response to therapy, we did not observe an inverse correlation between the expression of any these target genes and of miR-21-5p in our samples. Therefore, as miRNAs function can be tissue specific, we hypothesize that, in the context of rectal cancer samples, miR-21-5p might not be involved on the regulation of these target genes.

Finally, we have also performed functional experiments with two different cancer cell lines. We not only confirmed the regulation of SATB1 expression by miR21-5p but more importantly, we have confirmed that miR-21-5p and SATB1 play a direct role in response to in vitro chemoradiation.

\section{Conclusions}

We have identified miR-21-5p as a promising predictive marker for response to nCRT in rectal cancer patients. Patients with cCR based on very stringent criteria and high levels of miR-21-5p expression may be ideal candidates for alternative treatment strategies to radical surgery including a watch and wait approach. Further validation of these findings in larger cohorts may lead to the inclusion of this miRNA-based test in standard workup of patients prior to nCRT and help in clinical decision-making for patients with apparent cCR.

\section{Additional files}

Additional file 1: Table S1. Expression of miRNAs used for differential expression analyses among complete and incomplete responders. Samples from 1 to 14 represent complete responders patients, 15 to 39 represent incomplete responders patients, and 40 to 43 represent patients with early local recurrence. Normalized expression values are given in count per million (cpm).

Additional file 2: Figure S1. Evaluation of miR-21-5p by qPCR on primary tumor samples. Samples still available included 7 incomplete and 3 complete responders to nCRT. A colorectal cancer cell line (HCT116) was used as reference sample and the relative expression was calculated based on $\Delta \Delta C T$ method using miR-140-5p and miR-224-5p expression for normalization. Mann Whitney test performed. Figure S2. Evaluation of SATB1 gene expression by qPCR on primary tumor samples. Samples from 3 complete and 8 incomplete responders were used for SATB1 validation. A colorectal cancer cell line (HCT116) was used as reference sample and the relative expression was calculated based on $\Delta \Delta C T$ method using PUM1 and HMBS gene expression for normalization. Mann Whitney test performed.

Additional file 3: Tables S2. Patients clinical characteristics distribution according to miR-21-5p expression level (70,000 cpm cutoff established based on the training group). Table S4. Expression of miR-21-5p and 5 of its published target genes in rectal cancer samples. Pearson correlation was evaluated and only SATB1 gene presented a significant inversed correlation ( $r=-0.5, p=0.03$ )

Additional file 4: Table S3. Pearson correlation between the expression of miR-21-5p and all putative target genes in rectal cancer samples. Only SATB1 gene presented a significant inversed correlation $(r=-0.5$, $p=0.03)$.

\section{Competing interests}

The authors declare that they have no competing interests.

\section{Authors contributions}

CMLR, FB, FCK, PFA carried out sequencing and $\mathrm{gPCR}$ experiments. CMLR and FCK performed in vitro experiments with tumor cell lines. AHG, BSQ, NMF, JGR, ROP carried out sample collection and obtention of clinical data. CMLR, PAFG, RBP carried out sequencing and statistical analysis. CMLR, AHG, $J G R, A A C, R O P, R B P$ conceived the study and participated in its design. CMLR, AAC, ROP, RBP wrote the manuscript. All authors read and approved the final manuscript.

\section{Acknowledgements}

Financial support from So Paulo Research Foundation (FAPESP), grants: 2009/54349-9 and 2009/17785-5. The authors would like to thank Ceclia Maria Kalil Haddad, Gustavo Nader Marta and Pedro Ramos for their assistance in the irradiation of tumor cell lines.

\section{Author details}

${ }^{1}$ Centro de Oncologia Molecular, Hospital Srio-Libans, So Paulo, Brazil. ${ }^{2}$ Fundao Antnio Prudente, So Paulo, Brazil. $\quad{ }^{3}$ Angelita \& Joaquim Gama Institute, So Paulo, Brazil. ${ }^{4}$ University of So Paulo School of Medicine, So Paulo, Brazil. ${ }^{5}$ Ludwig Institute for Cancer Research, So Paulo, Brazil.

${ }^{6}$ Hospital Alemo Oswaldo Cruz, So Paulo, Brazil.

Received: 13 June 2014 Accepted: 28 November 2014

Published online: 11 December 2014

\section{References}

1. Kapiteijn E, Marijnen CA, Nagtegaal ID, Putter H, Steup WH, Wiggers T, Rutten HJ, Pahlman L, Glimelius B, van Krieken JH, Leer JW, van de Velde CJ: Preoperative radiotherapy combined with total mesorectal excision for resectable rectal cancer. N Engl J Med 2001, 345:638 646.

2. Minsky BD, Cohen AM, Kemeny N, Enker WE, Kelsen DP, Reichman B, Saltz L, Sigurdson ER, Frankel J: Combined modality therapy of rectal cancer: decreased acute toxicity with the preoperative approach. J Clin Oncol 1992, 10:1218 1224

3. Habr-Gama A, Perez RO, Kiss DR, Rawet V, Scanavini A, Santinho PM, Nadalin W: Preoperative chemoradiation therapy for low rectal cancer. Impact on downstaging and sphincter-saving operations. Hepatogastroenterology 2004, 51:1703 1707

4. Sanghera P, Wong DWY, McConkey CC, Geh J, Hartley A: Chemoradiotherapy for rectal cancer: an updated analysis of factors affecting pathological response. Clin Oncol (R Coll Radiol) 2008, 20:176 183.

5. Habr-Gama A, Perez RO, Nadalin W, Sabbaga J, Ribeiro U, Sousa AH S e, Campos FG, Kiss DR, Gama-Rodrigues J: Operative versus nonoperative treatment for stage 0 distal rectal cancer following chemoradiation therapy: long-term results. Ann Surg 2004, 240:711 717.

6. Habr-Gama A, Perez RO, Wynn G, Marks J, Kessler H, Gama-Rodrigues J: Complete clinical response after neoadjuvant chemoradiation therapy for distal rectal cancer: characterization of clinical and endoscopic findings for standardization. Dis Colon Rectum 2010, 53:1692 1698.

7. Bartel DP, Lee R, Feinbaum R: MicroRNAs: genomics, biogenesis, mechanism, and function. Cell 2004, 116:281 297. 
8. Croce CM: Causes and consequences of microRNA dysregulation in cancer. Nat Rev Genet 2009, 10:704 714

9. Calin GA, Croce CM: MicroRNA signatures in human cancers. Nat Rev Cancer 2006, 6:857 866.

10. Shenouda SK, Alahari SK: MicroRNA function in cancer: oncogene or a tumor suppressor? Cancer Metastasis Rev 2009, 28:369 378.

11. Nakajima G, Hayashi K, Xi Y, Kudo K, Uchida K, Takasaki K, Yamamoto M, Ju J: Non-coding MicroRNAs hsa-let-7 $g$ and hsa-miR-181b are associated with chemoresponse to S-1 in colon cancer. Cancer Genomics Proteomics 2006, 3:317 324

12. Svoboda M, Izakovicova Holla L, Sefr R, Vrtkova I, Kocakova I, Tichy B, Dvorak J: Micro-RNAs miR125b and miR137 are frequently upregulated in response to capecitabine chemoradiotherapy of rectal cancer. Int J Oncol 2008, 33:541 547.

13. Hummel R, Hussey DJ, Haier J: MicroRNAs: predictors and modifiers of chemo- and radiotherapy in different tumour types. Eur J Cancer 2010, 46:298 311.

14. Dworak O, Keilholz L, Hoffmann A: Pathological features of rectal cancer after preoperative radiochemotherapy. Int J Colorectal Dis 1997, 12:1923.

15. Kozomara A, Griffiths-Jones S: miRBase: integrating microRNA annotation and deep-sequencing data. Nucleic Acids Res 2011, 39:D152 D157.

16. Griffiths-Jones S, Grocock RJ, van Dongen S, Bateman A, Enright AJ: miRBase: microRNA sequences, targets and gene nomenclature. Nucleic Acids Res 2006, 34:D140 D144.

17. Robinson MD, McCarthy DJ, Smyth GK: edgeR: a Bioconductor package for differential expression analysis of digital gene expression data. Bioinformatics 2010, 26:139 140.

18. Gentleman RC, Carey VJ, Bates DM, Bolstad B, Dettling M, Dudoit S, Ellis B, Gautier L, Ge Y, Gentry J, Hornik K, Hothorn Y, Huber W, lacus S, Irizarry R, Leisch F, Li C, Maechler M, Rossini AJ, Sawitzki G, Smith C, Smyth G, Tierney $L$, Yang JYH, Zhang J: Bioconductor: open software development for computational biology and bioinformatics. Genome Biol 2004, 5:R80.

19. Benjamini $Y$, Hochberg $Y$ : Controlling the false discovery rate: a practical and powerful approach to multiple testing. J R Stat Soc B 1995, 57:289 300

20. Lewis $B P$, Burge $C B$, Bartel DP: Conserved seed pairing, often flanked by adenosines, indicates that thousands of human genes are microRNA targets. Cell 2005, 120:15 20.

21. Chang KH, Mestdagh P, Vandesompele J, Kerin MJ, Miller N: MicroRNA expression profiling to identify and validate reference genes for relative quantification in colorectal cancer. BMC Cancer 2010, 10:173.

22. Viprey VF, Corrias MV, Burchill SA: Identification of reference microRNAs and suitability of archived hemopoietic samples for robust microRNA expression profiling. Anal Biochem 2012, 421:566 572

23. Livak KJ, Schmittgen TD: Analysis of relative gene expression data using real-time quantitative PCR and the 2(-Delta Delta C(T)) Method. Methods San Diego Calif 2001, 25:402 408.

24. Han H-J, Russo J, Kohwi Y, Kohwi-Shigematsu T: SATB1 reprogrammes gene expression to promote breast tumour growth and metastasis. Nature 2008, 452:187 193.

25. Li Q-Q, Chen Z-Q, Xu J-D, Cao X-X, Chen Q, Liu X-P, Xu Z-D: Overexpression and involvement of special AT-rich sequence binding protein 1 in multidrug resistance in human breast carcinoma cells. Cancer Sci 2010, 101:80 86.

26. Meng W-J, Yan H, Zhou B, Zhang W, Kong $X-H$, Wang R, Zhan L, Li Y, Zhou Z-G, Sun X-F: Correlation of SATB1 overexpression with the progression of human rectal cancer. Int J Colorectal Dis 2012, 27:143 150

27. Tomimaru Y, Eguchi H, Nagano H, Wada H, Tomokuni A, Kobayashi S, Marubashi S, Takeda Y, Tanemura M, Umeshita K, Doki Y, Mori M: MicroRNA-21 induces resistance to the anti-tumour effect of interferon- $a / 5$-fluorouracil in hepatocellular carcinoma cells. $\mathrm{Br} J$ Cancer 2010, 103:1617 1626.

28. Deng J, Lei W, Fu J-C, Zhang L, Li J-H, Xiong J-P: Targeting miR-21 enhances the sensitivity of human colon cancer HT-29 cells to chemoradiotherapy in vitro. Biochem Biophys Res Commun 2014, 443:789 795.

29. Li Y, Zhao S, Zhen Y, Li Q, Teng L, Asai A, Kawamoto K: A miR-21 inhibitor enhances apoptosis and reduces $\mathrm{G}(2)-\mathrm{M}$ accumulation induced by ionizing radiation in human glioblastoma U251 cells. Brain Tumor Pathol 2011, 28:209 214.

30. Valeri N, Gasparini P, Braconi C, Paone A, Lovat F, Fabbri M, Sumani KM, Alder H, Amadori D, Patel T, Nuovo GJ, Fishel R, Croce CM: MicroRNA-21 induces resistance to 5 -fluorouracil by down-regulating human DNA MutS homolog 2 (hMSH2). Proc Natl Acad Sci U S A 2010, 107:21098 21103.

31. Feng Y-H, Wu C-L, Shiau A-L, Lee J-C, Chang J-G, Lu P-J, Tung C-L, Feng L-Y, Huang W-T, Tsao C-J: MicroRNA-21-mediated regulation of Sprouty2 protein expression enhances the cytotoxic effect of 5-fluorouracil and metformin in colon cancer cells. Int J Mol Med 2012, 29:920 926.

32. Beyer M, Thabet Y, Mller R-U, Sadlon T, Classen S, Lahl K, Basu S, Zhou X, Bailey-Bucktrout SL, Krebs W, Schnfeld EA, Bttcher J, Golovina T, Mayer CT, Hofmann A, Sommer D, Debey-Pascher S, Endl E, Limmer A, Hippen KL, Blazar BR, Balderas R, Quast T, Waha A, Mayer G, Famulok M, Knolle PA, Wickenhauser C, Kolanus W, Schermer B, et al: Repression of the genome organizer SATB1 in regulatory T cells is required for suppressive function and inhibition of effector differentiation. Nat Immunol 2011, 12:898 907.

33. Yang S, Banerjee S, de Freitas A, Cui H, Xie N, Abraham E, Liu G: miR-21 regulates chronic hypoxia-induced pulmonary vascular remodeling. Am J Physiol Lung Cell Mol Physiol 2012, 302:L521 L529.

34. Maas M, Beets-Tan RGH, Lambregts DMJ, Lammering G, Nelemans PJ, Engelen SME, van Dam RM, Jansen RLH, Sosef M, Leijtens JWA, Hulsew KWE, Buijsen J, Beets GL: Wait-and-see policy for clinical complete responders after chemoradiation for rectal cancer. J Clin Oncol 2011, 29:4633 4640.

35. Ghadimi BM, Grade M, Difilippantonio MJ, Varma S, Simon R, Montagna C, Fzesi L, Langer C, Becker H, Liersch T, Ried T: Effectiveness of gene expression profiling for response prediction of rectal adenocarcinomas to preoperative chemoradiotherapy. J Clin Oncol 2005, 23:1826 1838 .

36. Watanabe T, Komuro Y, Kiyomatsu T, Kanazawa T, Kazama Y, Tanaka J, Tanaka T, Yamamoto Y, Shirane M, Muto T, Nagawa H: Prediction of sensitivity of rectal cancer cells in response to preoperative radiotherapy by DNA microarray analysis of gene expression profiles. Cancer Res 2006, 66:3370 3374

37. Kim I-J, Lim S-B, Kang HC, Chang HJ, Ahn S-A, Park H-W, Jang S-G, Park J-H, Kim DY, Jung KH, Choi HS, Jeong S-Y, Sohn DK, Kim D-W, Park J-G: Microarray gene expression profiling for predicting complete response to preoperative chemoradiotherapy in patients with advanced rectal cancer. Dis Colon Rectum 2007, 50:1342 1353.

38. Rimkus C, Friederichs J, Boulesteix A-L, Theisen J, Mages J, Becker K, Nekarda $H$, Rosenberg R, Janssen K-P, Siewert JR: Microarray-based prediction of tumor response to neoadjuvant radiochemotherapy of patients with locally advanced rectal cancer. Clin Gastroenterol Hepatol 2008, 6:53 61 .

39. Nishioka M, Shimada M, Kurita N, Iwata T, Morimoto S, Yoshikawa K, Higashijima J, Miyatani T: Gene expression profile can predict pathological response to preoperative chemoradiotherapy in rectal cancer. Cancer Genomics Proteomics 2011, 8:87 92.

40. Kheirelseid EAH, Miller N, Chang KH, Curran C, Hennessey E, Sheehan M, Newell J, Lemetre C, Balls G, Kerin MJ: miRNA expressions in rectal cancer as predictors of response to neoadjuvant chemoradiation therapy. Int $J$ Colorectal Dis 2013, 28:247 260

41. Della Vittoria Scarpati G, Falcetta F, Carlomagno C, Ubezio P, Marchini S, De Stefano A, Singh VK, D Incalci M, De Placido S, Pepe S: A specific miRNA signature correlates with complete pathological response to neoadjuvant chemoradiotherapy in locally advanced rectal cancer. Int J Radiat Oncol Biol Phys 2012, 83:1113 1119 .

42. Svoboda M, Sana J, Fabian P, Kocakova I, Gombosova J, Nekvindova J, Radova L, Vyzula R, Slaby O: MicroRNA expression profile associated with response to neoadjuvant chemoradiotherapy in locally advanced rectal cancer patients. Radiat Oncol 2012, 7:195

43. Kalady MF, de Campos-Lobato LF, Stocchi L, Geisler DP, Dietz D, Lavery IC, Fazio WW: Predictive factors of pathologic complete response after neoadjuvant chemoradiation for rectal cancer. Ann Surg 2009, 250:582 589.

44. Yamamichi N, Shimomura R, Inada K, Sakurai K, Haraguchi T, Ozaki Y, Fujita S, Mizutani T, Furukawa C, Fujishiro M, Ichinose M, Shiogama K, Tsutsumi Y, Omata M, Iba H: Locked nucleic acid in situ hybridization analysis of miR-21 expression during colorectal cancer development. Clin Cancer Res 2009, 15:4009 4016

45. Chan JA, Krichevsky AM, Kosik KS: MicroRNA-21 is an antiapoptotic factor in human glioblastoma cells. Cancer Res 2005, 65:6029 6033.

46. Ren J, Zhu D, Liu M, Sun Y, Tian L: Downregulation of miR-21 modulates Ras expression to promote apoptosis and suppress invasion of Laryngeal squamous cell carcinoma. Eur J Cancer 2010, 46:3409 3416.

47. Wu CW, Ng SSM, Dong YJ, Ng SC, Leung WW, Lee CW, Wong YN, Chan FKL, Yu J, Sung JJY: Detection of miR-92a and miR-21 in stool samples as 
potential screening biomarkers for colorectal cancer and polyps. Gut 2012, 61:739 745.

48. Toiyama Y, Takahashi M, Hur K, Nagasaka T, Tanaka K, Inoue Y, Kusunoki M, Boland CR, Goel A: Serum miR-21 as a Diagnostic and Prognostic Biomarker in Colorectal Cancer. J Natl Cancer Inst 2013, 105:849 859.

49. Xia X, Yang B, Zhai X, Liu X, Shen K, Wu Z, Cai J: Prognostic role of microRNA-21 in colorectal cancer: a meta-analysis. PLoS One 2013, 8:e80426.

50. Yu Y, Sarkar FH, Majumdar APN: Down-regulation of miR-21 induces differentiation of chemoresistant colon cancer cells and enhances susceptibility to therapeutic regimens. Transl Oncol 2013, 6:180 186.

51. Schetter AJ, Leung SY, Sohn JJ, Zanetti KA, Bowman ED, Yanaihara N, Yuen ST, Chan TL, Kwong DLW, Au GKH, Liu C-G, Calin GA, Croce CM, Harris CC: MicroRNA expression profiles associated with prognosis and therapeutic outcome in colon adenocarcinoma. JAMA 2008, 299:425 436.

52. Drebber U, Lay M, Wedemeyer I, Vallbhmer D, Bollschweiler E, Brabender J, Mnig SP, Hlscher AH, Dienes HP, Odenthal M: Altered levels of the onco-microRNA 21 and the tumor-supressor microRNAs 143 and 145 in advanced rectal cancer indicate successful neoadjuvant chemoradiotherapy. Int J Oncol 2011, 39:409 415.

53. Sun F, Lu X, Li H, Peng Z, Wu K, Wang G, Tong Q: Special AT-rich sequence binding protein 1 regulates the multidrug resistance and invasion of human gastric cancer cells. Oncol Lett 2012, 4:156 162.

54. Han B, Luan L, Xu Z, Wu B: Expression and biological roles of SATB1 in human bladder cancer. Tumour Biol 2013, 34:2943 2949.

doi:10.1186/s12920-014-0068-7

Cite this article as: Lopes-Ramos et al.: Overexpression of miR-21-5p as a predictive marker for complete tumor regression to neoadjuvant chemoradiotherapy in rectal cancer patients. BMC Medical Genomics 2014 7:68.

\section{Submit your next manuscript to BioMed Central and take full advantage of:}

$\bowtie$ Convenient online submission

$\otimes$ Thorough peer review

$\nabla$ No space constraints or color $\nabla$ gure charges

$\triangle$ Immediate publication on acceptance

\Inclusion in PubMed, CAS, Scopus and Google Scholar

$\otimes$ Research which is freely available for redistribution 